\title{
Conceptual Framework for the Conservation of Natural Environment from Toxic Ionic Liquids by QSAR Model
}

\author{
Muhammad Ishaq Khan ${ }^{1}$ Dzulkarnain Zaini ${ }^{1 *}$, and Azmi Mohd Shariff ${ }^{1}$ \\ ${ }^{1}$ Center of Advance Process Safety (CAPS) Universiti Teknologi PETRONAS Malaysia
}

\begin{abstract}
The natural environment has been affected by human activities to fulfil daily life needs. Abundance and hazardousness of the chemicals including ionic liquids is one of the most challenging aspect to be handled by human as well as for the natural environment. Due to ionic structure, ionic liquids are very good choice for a variety of applications. The natural environment might be affected by the ionic liquids which can be toxic. Therefore, there is a need to address this problem by studying the ecotoxicological behaviour of these ionic liquids. The main objective of current research is to model the toxicity ecotoxicological behaviour is studied by quantitative structure activity relationship (QSAR). QSARs predicts the toxicity of ionic liquids. In current research a relationship between polarizability and toxicity for imidazolium ionic liquids with different alky chain length having NTF2 anion has been modelled. The success of current research will be very helpful to protect the nature by minimizing the killing of testing animals as well as ensuring the safety of biotic components of the ecosystem.
\end{abstract}

\section{Introduction}

The environment is the total of all surroundings of a living organism, including natural forces and other living things. There are three types of environments; Natural environment, built environment and the social environment [1]. The natural environment is of great importance because any disturbance in this environment will break down the balance of all other environments. The natural environment is composed of naturally occurring living and non-living things. The main components of the natural environment are living organisms, water, air, and land. Disturbance in natural environmental cause disturbance in built environment and social environment. Therefore, it is very much important to keep natural environment protected. Seas and oceans are amongst the best sources of human needs such as the economy, food, and other many other environmental benefits. Thus, the protection of these water sources must be ensured to save the economy, food and cultural benefits as the need of humanity and for the sake of preservation of nature.

Use of Ionic Liquids (ILs) for the processing and purification of natural gas and many other industrial processes is one of the hot research topics nowadays [2]. An extensive research is being conducted to utilize ionic liquids in industries because of the salient physical and chemical characteristics of these liquids. Ionic liquids are very useful liquids. Due to ionic structure, several different combinations of cations, anions, and other replaceable constituents make these liquids a very good choice for a variety of applications [3]. ILs are usually considered as green solvents but until last decade the toxicity of ILs had become a hot issue.

Our natural environment and entire ecosystem might be affected by the toxic ionic liquids. It is necessary to evaluate the ecotoxicological risks associated with ILs so that natural environment could be protected from the hazardous of toxic ILs. Quantitative Structure-Activity Relationship (QSAR) is one of the techniques that can be used for the ecotoxicological assessment of ionic liquids which may help to stop killing of testing animals and provide the basis for synthesizing new environmentally friendly liquids. QSAR modelling helps to develop a mathematical correlation between the chemical features and the behaviour of the compounds with same chemical structures. QSAR technique enables to explore the characteristics of a chemical in the form of encoded descriptors. Descriptors are in the numerical form which represents the properties of the liquids and helps in developing mathematical correlation. These are the descriptors which are helpful in modification or finetuning the behaviour of the chemicals under the consideration into desirable one by appropriate changes in chemical structures[4,5]. QSAR is used by a few researchers for ecotoxicological risk assessment of ionic liquids [6]. A relationship between the structure and the toxicity was developed by a QSAR model which indicated that with the increase of several oxygen atoms present in ILs caused to decrease the toxicity of those ILs to rat cell[7]. In other hand, QSAR has been used by a larger number of the researchers working on the ecotoxicology of ILs [5, 8-10].

\footnotetext{
* Corresponding author: dzulkarnain.zaini@utp.edu.my
} 


\section{Methodology}

The complete conceptual framework for this ongoing research is presented in Figure 1. Following are the steps completely describing the methodology for the current work : -

A) Comprehensive literature review on laboratory testing the toxic samples of ILs : (i) Ecotoxicity studies of ionic liquids (ii) Selection criteria for designing of greener ILs

B) QSAR modelling followed by : (i) Molecular studies of ILs (ii) Selection of molecular descriptors (input for model) (iii) Development of correlation between descriptor and toxicity (development of QSAR model)

C) Validation of models by prioritizing/designing of ILs followed by acute toxicity experimental selected ILs

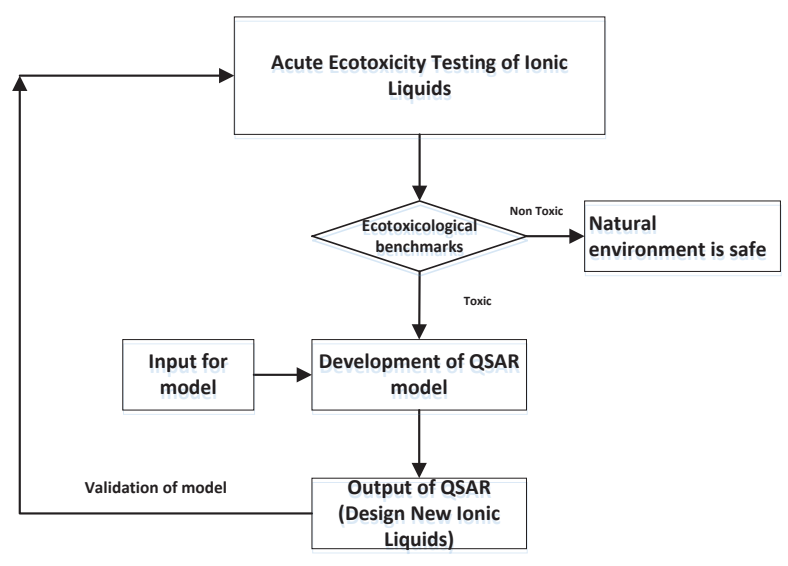

Fig. 1. Methodological framework of the research

\subsection{Ecotoxicity Measurement of ILs}

For the quantification of the toxicity hazard of the chemicals to the species which we desire to protect in exposed ecosystem, the risk assessors use laboratory effect value, i.e., is the concentration of a substance yielding an observable and measurable effect to a population (i.e., of a single type) of species. Ecotoxicological studies for aquatic ecosystem are generally not studied for a prolonged period because long term studies are more expensive to conduct relative to short-term studies. Furthermore, there is no data available for chronic effects of ionic liquids. Only shortterm data is available. EC50 is the concentration at which $50 \%$ of the test species are affected. In this research new novel QSAR will predict the ionic liquids toxicity as a function of the polarity of the ionic liquid composed of different cations, anions, the functional groups, and side alkyl chains.

\subsection{Quantitative Structure Activity Relationship (QSAR)}

Quantitative structure-activity relationship (QSAR) model predicts the response of liquids by using structural information using mathematical correlations. QSAR are the statistical techniques which do not involve any physical experiments to test the toxicity of liquids, rather it predicts toxicities based on the relationship between toxicity and chemical composition of liquids. Importance of QSAR has been proven where the killing of the testing animals is involved. Hence QSAR modelling is a lifesaving technique for the testing animals. In this work, we have selected toxicity[11] and polarizability data from the literature[12]. A small data set of three ionic liquids was used. Two descriptors alkyl chain length and polarizability were used to model the toxicity of imidazolium ionic liquids with NTf2 anion as shown in Table 1.

Table 1. Toxicity and polarizability of Ionic Liquids

\begin{tabular}{|cccc|}
\hline Ionic Liquids & $\begin{array}{c}\text { No. of } \\
\text { Carbon }\end{array}$ & Polarizability & EC50 \\
\hline [C2C1im][NTf2] & 2 & 0.98 & 330.23 \\
{$[$ C6C1im][NTf2] } & 6 & 0.97 & 22.8 \\
{$[\mathrm{C} 8 \mathrm{C} 1$ im] $]$ NTf2] } & 8 & 0.96 & 6.44 \\
\hline
\end{tabular}

\section{Results and Discussion}

Several research studies have been carried out to observe the main driver of toxicity of ionic liquids. Alkyl chain length is one of the major contributors to the toxicity of ionic liquids. Longer the chain length of alkyl constituent, higher is the toxicity. This trend is widely accepted and commonly found in most of the ionic liquids. There is a need to understand why alkyl chain length have been proven to be the main driver of toxicity of ILs [13]. Structural parameter of ILs is considered as one of the reasons for their toxic behaviour. For example, we have found that toxicity of ILs is related to the polarity, as polarity and alkyl chain length are directly related. A graph is plotted between alkyl chain length and the polarizability of the imidazolium ILs with NTF2 anion in and polarizability and EC50 in Fig 2.

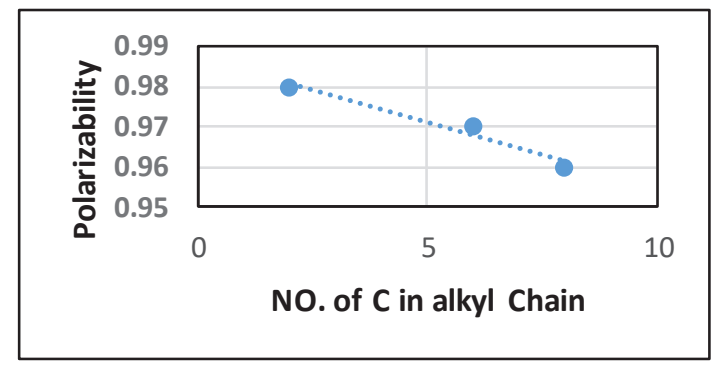

Fig. 1 Alkyl chain length vs Polarizability of ILs 


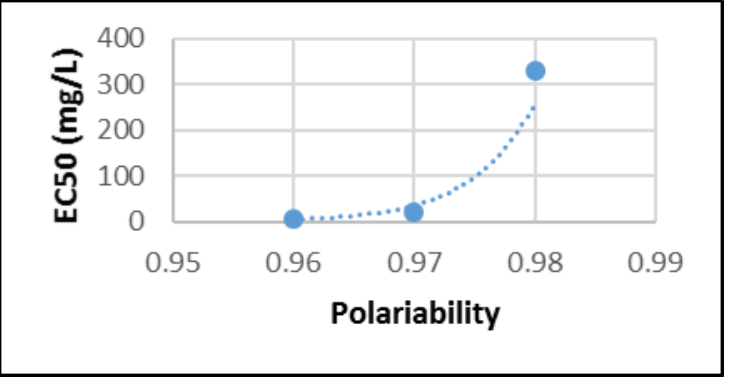

Fig. 2 Polarizability vs EC50 of ILs

Fig. 2 shows direct relationship between the polarizability and the alkyl chain length of imidazolium ionic liquids with NTF2 anion while Fig. 3 shows inverse relationship between the polarizability and EC50 of imidazolium ionic liquids with NTF2 anion. Increasing EC50 means decreasing toxic behaviour so the polarizability has direct relationship with toxicity. QSAR model is developed which predicted the toxicity based on two descriptors i.e., polarizability of the ILs and the length of alkyl chain. In our research we proved that polarizability is the one property of ILs which contributed to increase of toxicity. Multiple linear regression was performed by using Microsoft excel. The regression is resulted into a model equation (1).

$$
y=27542.88-145.535 \times 1-27471 \times 2
$$

where $\mathrm{y}$ is the toxicity of ionic liquids in $\mathrm{mg} / \mathrm{L}$ and $\mathrm{x} 1$ is the length of alkyl chain in the form of the number of $\mathrm{C}$ in the alkyl chain and $\mathrm{x} 2$ is the polarizability of imidazolium ILs studied.

\subsection{Validation of The Model}

Although this QSAR model is developed for a small set of data, however, it has provided an idea to relate the toxicity as a function of polarizability hence polarity. This is a simple short model describing the toxicity as a function of polarizability and alkyl chain length of imidazolium ILs with the NTf2 anion. When alkyl chain length attached with the cation of ionic liquids is increased, the polarizability of the whole compound is decreased. As a result of polarity is increased. Increased polarity indicates the solubility towards water[14].

A linear relationship is found between alky chain length and polarizability [15]. Only limited literature data are found in literature which can be compared with our QSAR model[16]. Currently we have a small data on polarity of ILs from literature [17] [18]. Toxicity data is taken from experimental studies of ionic liquids toxicity on vibrio fischeri by Luis et al.[19].
Table 2. Polarizability and alkyl chain length in ILs

\begin{tabular}{|cccc|}
\hline $\begin{array}{c}\text { Ionic } \\
\text { Liquids }\end{array}$ & $\begin{array}{c}\text { Length of alkyl } \\
\text { Chain }\end{array}$ & Polarizability & EC50 \\
\hline BMIM & & 11.7 & 2137 \\
BF4 & 4 & 11.3 & 1513 \\
HMIM & & & \\
BF4 & 6 & 7.5 & 257 \\
OMIM & & & \\
BF4 & 8 & &
\end{tabular}

From above table we see that with increase in length of alkyl chain polarizability is decreasing with is follows the trend set up in our model.



Fig. 3 Alkyl chain length and EC50 for validation of model

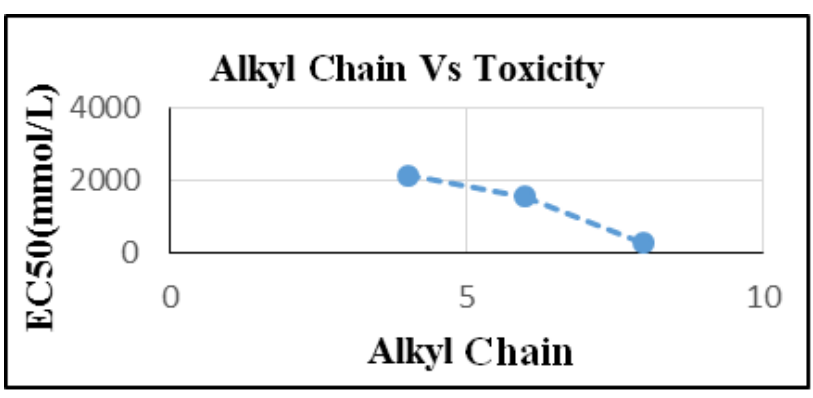

Fig. 4 Alkyl chain length and EC50 from modeling data

From Fig. 4 and 5, we can see that our model is successfully validated as trend of alkyl chain length vs toxicity is same as predicted by model.

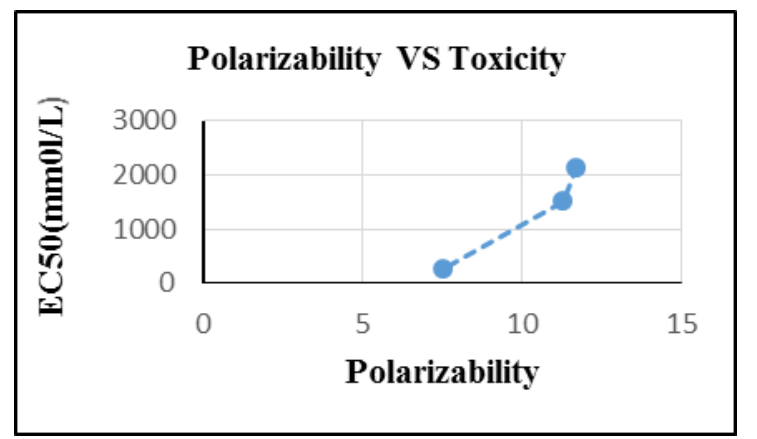

Fig. 6 Alkyl chain length and EC50 from validation of model 


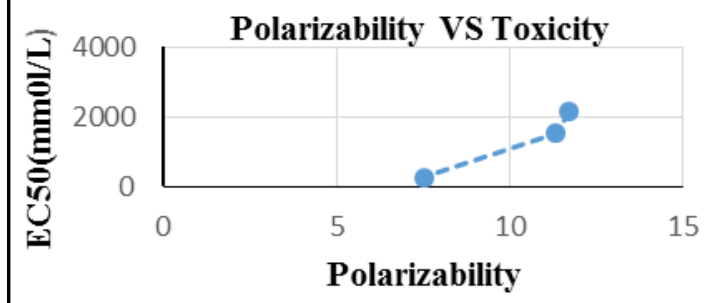

Fig. 7 Alkyl Polarizability and EC50 from modeling data

From figure 6 and 7, same trend in polarizability and toxicity is observed which successfully validates QSAR model in current study.

\section{Conclusion}

This research will provide a new technique to design greener ionic liquids which will be friendly to the biotic components of the ecosystem. QSAR predict the toxicity of new untested ionic liquids, without doing experiments, based on their chemical compositions, cations-anions, and functional group interactions. This research will be very helpful to design alternative ionic liquids for sustainable chemical operations resulting in no negative impacts on the environment, especially on the aquatic ecosystem. Correlation of polarizability with toxicity and alkyl chain length will provide detailed information to understand the toxicology of the ionic liquids. As a result of polarity is increased, it indicates the solubility towards water and water solubility of ionic liquids causes ILs to enter the aquatic life and other living species. In-depth knowledge of the intermolecular attraction in ILs leads to a better understanding of the behaviour of these liquids, especially the toxicity mechanism. As ILs are new liquids having no major potential industrial applications, it must be ensured that synthesis of new potential ILs should be carried out carefully and by keeping in mind the ecotoxicological effects of these liquids. Better understanding of the toxicological trends of ILs can lead to the design of green and inherently safer ILs by developing and applying QSAR model will reduce the killing of the testing animal, hence nature will be protected.

\section{References}

1. Wells, N.M., G.W. Evans, and K.A. Cheek, Environmental psychology. Environmental Health: From Global to Local, 2016: p. 203.

2. Qiao, Y., et al., Temperature-Responsive Ionic Liquids: Fundamental Behaviours and Catalytic Applications. Chemical Reviews, 2017.

3. Rodríguez, H., Ionic Liquids in the Context of Separation Processes, in Ionic Liquids for Better Separation Processes. 2016, Springer. p. $1-9$.
4. Roy, K., S. Kar, and R.N. Das, Understanding the basics of QSAR for applications in pharmaceutical sciences and risk assessment. 2015: Academic press.

5. Roy, K., R.N. Das, and P.L. Popelier, Predictive QSAR modelling of algal toxicity of ionic liquids and its interspecies correlation with Daphnia toxicity. Environmental Science and Pollution Research, 2015. 22(9): p. 6634-6641.

6. Khan, M.I., et al., Framework for Ecotoxicological Risk Assessment of Ionic Liquids. Procedia Engineering, 2016. 148: p. 1141-1148.

7. Zhao, Y., et al., Toxicity of ionic liquids: database and prediction via quantitative structure-activity relationship method. Journal of hazardous materials, 2014. 278: p. 320-329.

8. Ghanem, O.B., et al., Effect of imidazoliumbased ionic liquids on bacterial growth inhibition investigated via experimental and QSAR modelling studies. Journal of hazardous materials, 2015. 297: p. 198-206.

9. Wang, C., et al., Assessment of bromide-based ionic liquid toxicity toward aquatic organisms and QSAR analysis. Ecotoxicology and environmental safety, 2015. 115: p. 112-118.

10. Peric, B., et al., Quantitative structure-activity relationship (QSAR) prediction of (eco) toxicity of short aliphatic protic ionic liquids. Ecotoxicology and environmental safety, 2015. 115: p. 257-262.

11. Ab Rani, M., et al., Erratum: Understanding the polarity of ionic liquids (Physical Chemistry Chemical Physics (2011) DOI: 10.1039/c1cp21262a). Physical Chemistry Chemical Physics, 2011. 13(48): p. 21653.

12. Ventura, S.P., et al., Designing ionic liquids: the chemical structure role in the toxicity. Ecotoxicology, 2013. 22(1): p. 1-12.

13. Bubalo, M.C., et al., Imidiazolium based ionic liquids: effects of different anions and alkyl chains lengths on the barley seedlings. Ecotoxicology and environmental safety, 2014. 101: p. 116-123.

14. Kurnia, K.A., et al., Comprehensive study on the impact of the cation alkyl side chain length on the solubility of water in ionic liquids. Journal of molecular liquids, 2015. 210: p. 264271.

15. Ab Rani, M., et al., Understanding the polarity of ionic liquids. Physical Chemistry Chemical Physics, 2011. 13(37): p. 16831-16840.

16. Krishnan, S., et al. Biodegradability of immidazolium, pyridinium, piperidinium and pyrrolidinium based ionic liquid in different water source. in AIP Conference Proceedings. 2016. AIP Publishing. 
17. Weingärtner, H., The static dielectric constant of ionic liquids. Zeitschrift für Physikalische Chemie, 2006. 220(10): p. 1395-1405.

18. Singh, T. and A. Kumar, Static dielectric constant of room temperature ionic liquids: internal pressure and cohesive energy density approach. The Journal of Physical Chemistry B, 2008. 112(41): p. 12968-12972.

19. Luis, P., A. Garea, and A. Irabien, Quantitative structure-activity relationships (QSARs) to estimate ionic liquids ecotoxicity EC 50 (Vibrio fischeri). Journal of Molecular Liquids, 2010. 152(1): p. 28-33. 